\title{
Morphology and Structure of Compacted Coated Powders Studied by SEM and TEM
}

F. Simões, ${ }^{*} * *$ and B. Trindade, ${ }^{* *}$

* ISEC, Mechanical Department, Engineering Institute of Coimbra, Rua Pedro Nunes, Coimbra 3030-199, Portugal.fsimoes@isec.pt

** CEMUC, Mechanical Department of Coimbra University, Coimbra 3030-788, Portugal.

The interest in titanium based intermetallics, such as Ti-Al alloys, for high temperatures applications has been increased in recent years mainly due to their high plastic deformation resistance, chemistry stability (oxidation and corrosion resistance) and creep and fatigue resistance at high temperatures. However, the industrial application of Ti-Al intermetallics is very limited so far, due to the lack of ductility and fracture toughness at room temperature with associated processing difficulties [1]. To overcome this problem and to improve mechanical properties of Ti$\mathrm{Al}$ intermetallics, a powder mixture with atomic composition of $\mathrm{Ti}_{52} \mathrm{Al}_{48}$ was synthesized by mechanical alloying (MA) and subsequently coated with a ductile element (aluminium), by d.c. magnetron sputtering [2]. Later, in order to obtain a compacted material, as final step, the coated MA'ed powders were submitted to hot isostatic pressing (HIP), giving rise to a bulk material. The aim of the current work is to understand the influence of coating layer in mechanical alloyed (MA'ed) powders and the influence in bulk material properties.

According to the ductility of the elemental powders, one may consider that $\mathrm{Ti}_{52} \mathrm{Al}_{48}$ is a ductileductile system. In fact, as showed in figure 1 , in an initial milling stage, the particles are flattened and the particles size increase as result of welding predominance. This stage is followed by a continuous decrease of particles size, corresponding to the fracture/welding stationary period. At 50 $\mathrm{h}$ of milling, a steady-state equilibrium is attained, where the particles reach approximately a round shape.

Concerning to the coating process, atoms of aluminium target are sputtered towards powder particles and are deposited as thin surrounding films. The surface area of the powders exposed to the sputtering plasma is continuously renovated by translation and vibration movements of powders tray, which improves the efficiency of this process. As reported by Kersten et al [3] and Fernandes et al [4] Al-coated powders show a rough and cauliflower-like shape (fig. 2a), which make them attractive for several applications. The cross section analysis (fig. 2b) enables to identify the powders core and a non-uniform coating layer, with a maximum thickness of $2 \mu \mathrm{m}$, measured in SEM micrographs.

The coated MA'ed powders, were cold isostatic pressed, encapsulated, degassed, sealed and submitted to HIP cycle (150 MPa at $900{ }^{\circ} \mathrm{C}$, during $2 \mathrm{~h}$ ). In the SEM images of cross-section compacted powders (fig. 3 ) is distinguish the coating and the powders core that give rise to the bulk and it is possible to say that the selected HIP parameters led to a good consolidation level. The low porosity reached during compaction of compounds with different strain abilities, is succeed mainly due to the deformation of softer compound, as reported by Pagounis et al [5]. In the present study, it is observed a similar situation, once the hardened powders core has no significant deformation during compaction process, while the Al-coating, due to the high plasticity, flows among particles, 
filling the open porosities, reaching a more uniform coating layer. The Energy Dispersive Spectroscopy (EDS) analysis performed in the coating layer and in powders core confirms that $\mathrm{Al}$ and $\mathrm{Ti}$ are the major elements present in the bulk material. Despite of aluminium richer interface, $\mathrm{Ti}$ is detected in coating, meaning that during hot isostatic pressing, diffusion of this element occurred from the powders to the coating.

SEM image of compacted sample and corresponding qualitative elementary analysis of titanium, aluminium and oxygen was determined by electron prove microanalysis (EPMA). The results are presented in figure 4. While titanium and aluminium are spread all over bulk material, oxygen content is higher around the particles (blue regions) and lower in core (purple regions). This means that the structure existing before the consolidation process plays a significant role on the distribution of oxygen contamination level. In fact, it is likely that before heating, during consolidation, the structure of powders core migh be formed by Ti-Al intermetallics as result of mechanical alloying process [6] and, therefore, with lower affinity to the oxygen than Al-coating [1].

The grain size determined in powders core by transmission electron microscopy is about $300 \mathrm{~nm}$, where is possible to identify grains in a range of $200-400 \mathrm{~nm}$ (fig. 5a). As observed in figure 5b, is often observed twinning in TEM samples, meaning that this mechanism deformation has an important role during consolidation. Besides, precipitates around the central twinned grain can be observed. Pontual EDS from precipitates shows a Ti-richer phase that might be $\alpha_{2}-\mathrm{Ti}_{3} \mathrm{Al}$ phase, whereas X-ray diffraction made in $\mathrm{MA}^{\prime}$ ed powders in a prior study [7], had identified a mixture of $\gamma$-TiAl $+\alpha_{2}-\mathrm{Ti}_{3} \mathrm{Al}$ phases, where $\alpha_{2}-\mathrm{Ti}_{3} \mathrm{Al}$ is the minor phase. The diffraction pattern made in TEM sample (fig. 5c) confirms that the main phase present in powders core is $\gamma$-TiAl.

As conclusion, it is possible to say that SEM and TEM were useful techniques that allowed understanding the morphology and structure of a compacted sample, obtained starting from mechanically alloyed $\mathrm{Ti}_{52} \mathrm{Al}_{48}$ powders, coated with aluminium.

Acknowledgements

The authors gratefully acknowledge the financial support from Portuguese Foundation for Science and Technology (FCT) through project POCTI/CTM/46498/2002.

\section{References}

[1] Gerhard Sauthoff, Intermetallics - Chapter 3, VCH, Germany, 1995.

[2] F. Simões and B. Trindade, Mater. Sci. Eng., A 397 (2005) 257.

[3] H. Kersten, P. Schmetz and G. M. W. Kroesen, Surf. Coat. Technol. (1998) 108.

[4] C. M. Fernandes, A. M. R. Senos and M. T. Vieira, Mat. Sci. Forum 455-456 (2004) 295.

[5] E. Pagounis, M. Talvitie and V. K. Lindroos, Mat. Res. Bull. 31-10 (1996) 1277.

[6] D. L. Zhang and D. Y. Ying, Mat. Sci. Forum 360 (2001) 323.

[7] F. Simões, B. Trindade and J. A. Santos, Mat. Sci. Forum 455-456 (2004) 285. 


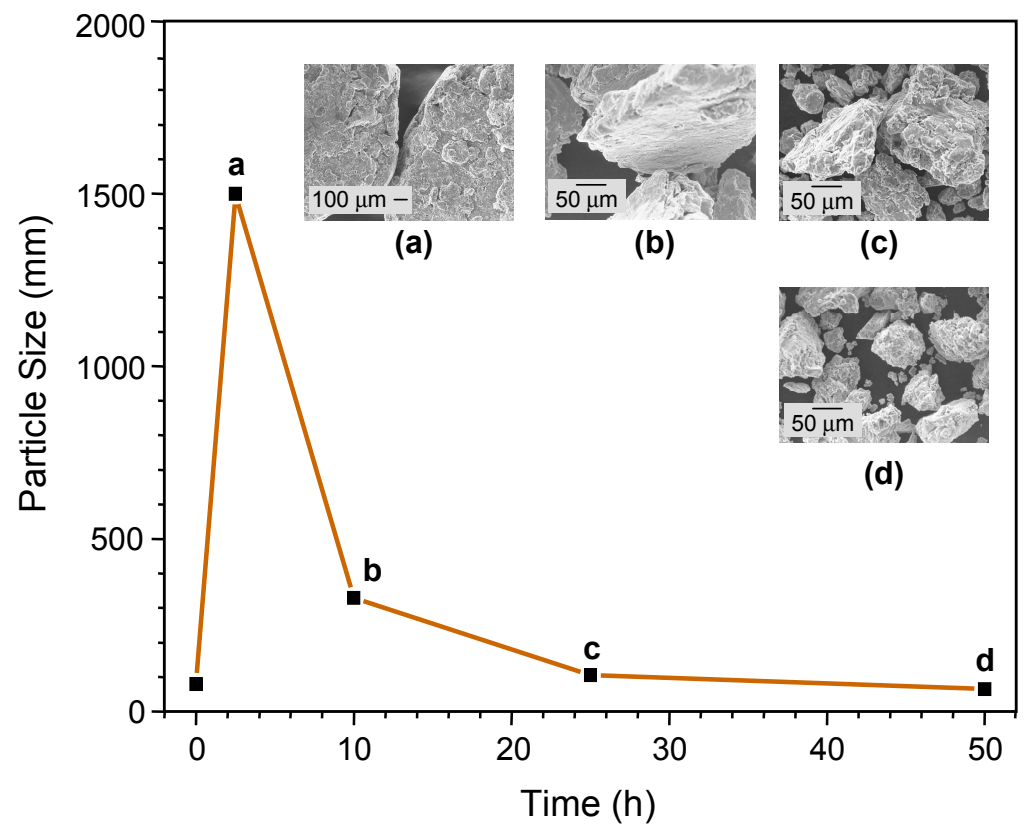

Figure 1. Morphology and mean value of particles size of $\mathrm{Ti}_{52} \mathrm{Al}_{48}$ mixture, at different milling times, during mechanical alloying up to $50 \mathrm{~h}$.

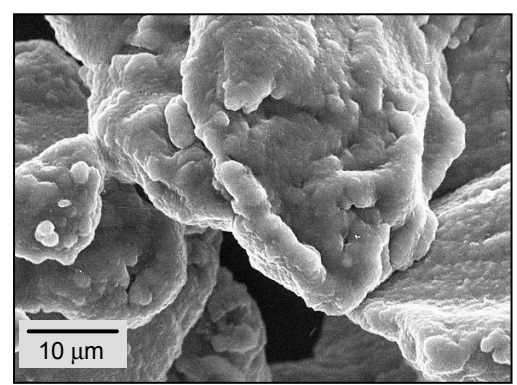

(a)

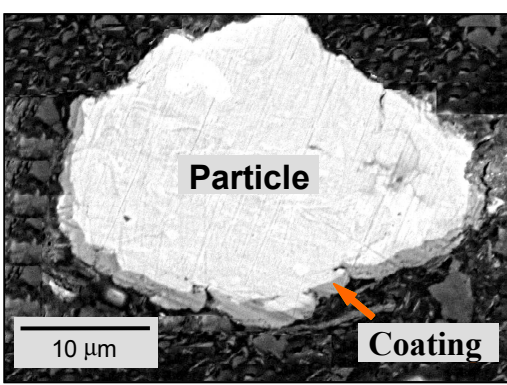

(b)

Figure 2. SEM images of $\mathrm{Ti}_{52} \mathrm{Al}_{48}$ mechanically alloyed powders coated with $\mathrm{Al}$.

(a) Surface morphology; (b) cross section coated particle embedded in thermosetting polymer. 

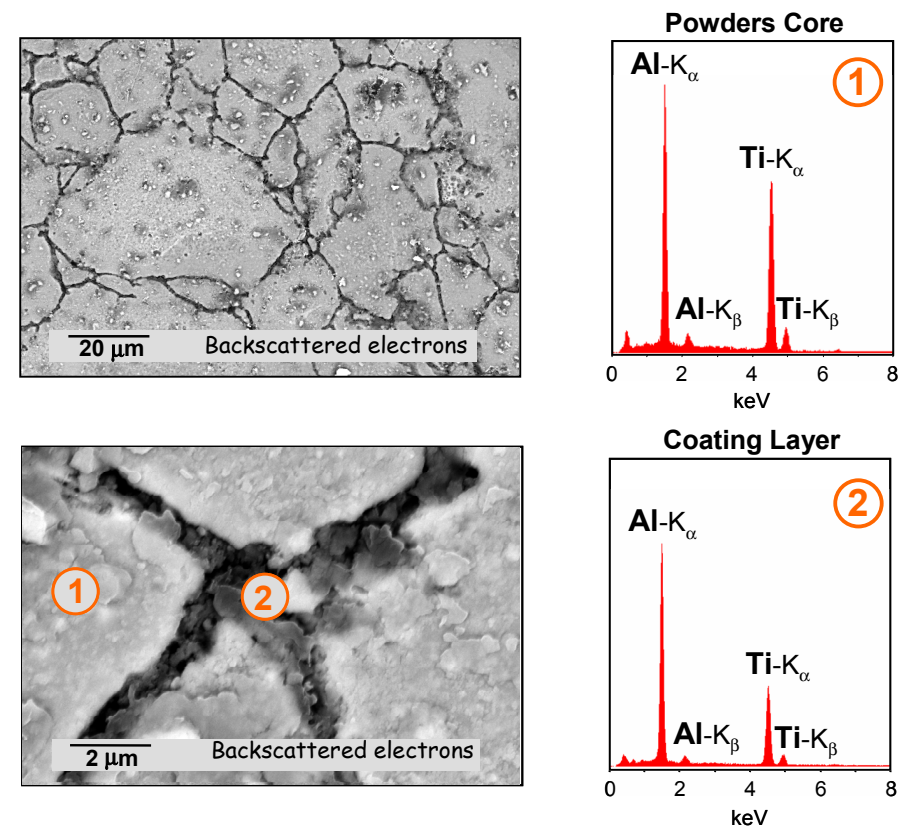

Figure 3. SEM images at different magnification of bulk material and corresponding EDS spectra made in powders core and coating layer.

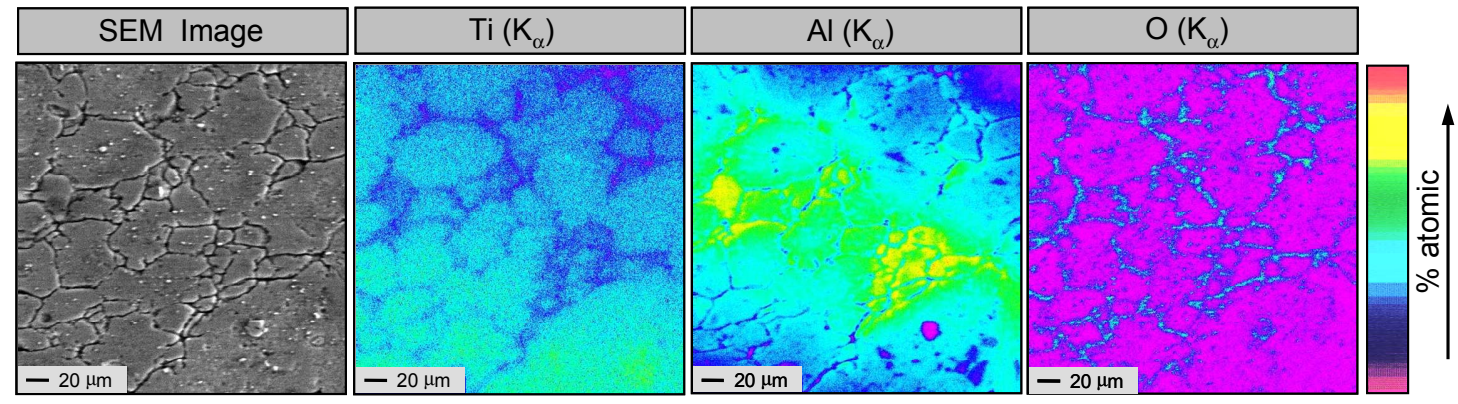

Figure 4. SEM image and corresponding EPMA analysis of titanium, aluminium and oxygen elementary distribution obtained in bulk material.

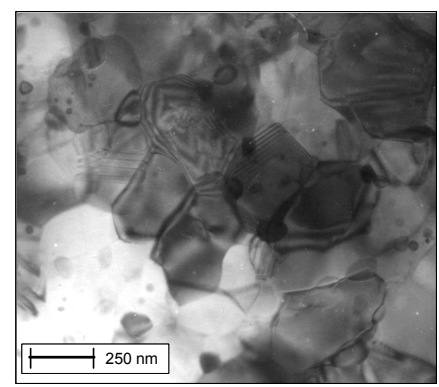

(a)

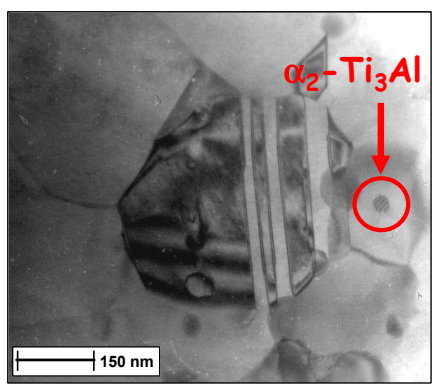

(b)

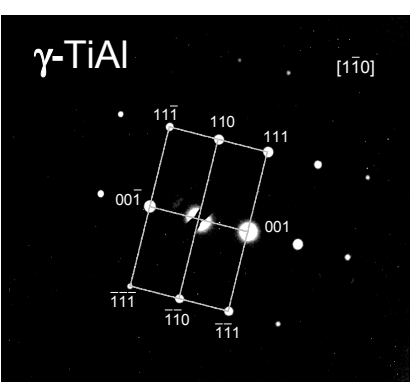

(c)

Figure 5. TEM results of bulk material, obtained on powders core. 